\title{
MODELING THE RELATIONSHIP BETWEEN WEATHER PARAMETERS AND CHOLERA IN THE CITY OF MAROUA, FAR NORTH REGION, CAMEROON
}

\author{
Mouhaman ARABI ${ }^{1}$, Moise Chi NGWA ${ }^{2}$
}

DOI: 10.21163/GT_2019.141.01

\begin{abstract}
:
Locally measured variables such as temperature and rainfall have been positively associated with increased cholera incidence in multiple studies. The study examined the effect of rainfall, temperature and relative humidity on cholera incidence in the City of Maroua, located in the Far North of Cameroon. The relative individual contribution of rainfall, temperature and humidity and then their combined contribution on the occurrence of cholera were examined. Using monthly time series of cholera epidemiological data, average monthly rainfall $(\mathrm{mm})$, average monthly air temperature (degree Celsius), and average monthly relative humidity (percent) data from 1996 to 2011.We implemented Generalized Additive Modeling (GAM) procedures to measure the contribution of each weather parameter to the incidence of cholera and identified the most influential parameter on cholera incidence. We found that taken individually, rainfall, temperature and humidity are correlated with cholera incidence but temperature seems more determinant since it has a higher deviance explained (26\%). Furthermore, the association between temperature and rainfall in the multivariate model with interaction has the highest deviance explained is $64.4 \%$, the lowest AIC is 1911.963, and the highest $\mathrm{R}^{2}$ (0.6). These results indicate that statistical time series models in general and the Generalized Additive models in particular should lead to a better understanding of the disease mechanism that can assist in the planning of public health interventions. These results contribute also to the growing debate on climate and cholera.
\end{abstract}

Key-words: Cholera, Maroua, Cameroon, GAM model.

\section{INTRODUCTION}

Cholera is an acute diarrheal infection caused by ingestion of food or water contaminated with the bacterium Vibrio cholerae. Cholera remains a global threat to public health and an indicator of inequity and lack of social development. Researchers have estimated that every year, there are roughly 1.3 to 4.0 million cases, and 21000 to 143000 deaths worldwide due to cholera (Ali et al., 2015). The disease which has a short incubation period of two hours to five days and this enhances the potentially explosive pattern of cholera outbreaks (WHO, 2012) has been scrutinized since the birth of epidemiology, and it is still a subject of intense interest for modern-day epidemiologists (Codeço \& Coelho, 2006). During the $19^{\text {th }}$ century, cholera spread across the world from its original reservoir in the Ganges delta in India. Seven cholera pandemics have killed millions of people across all continents with the seventh pandemic that began in 1961 in the Celebes, Indonesia,

\footnotetext{
${ }^{1}$ University of Maroua, Advanced School of Engineering, Department of Environmental Sciences, P.O. Box: 46 Maroua, Cameroon; mouhamanarabi@hotmail.com

${ }^{2}$ Johns Hopkins Bloomberg School of Public Health, Department of International Health, 21205, Baltimore, Maryland,USA; mngwa2@jhu.edu
} 
currently ongoing. Recently, its re-emergence has been noted in parallel with the everincreasing size of vulnerable populations living in unsanitary conditions, such as peri-urban slums, as well as camps for internally displaced people or refugees, where minimum requirements of clean water, sanitation, and hygiene are not met (WHO, 2012).

Cholera has a strong relationship with weather events (Lipp et al., 2002) but displays a complex relationship to extremes of weather conditions (McMichael, 2015). Locally measured variables such as temperature and rainfall have been positively associated with increased cholera incidence in multiple studies (Reyburn et al., 2011). The relationship between climate and cholera has been a subject of study for a very long time. However, during the past 2 decades, development of modern tools and technologies has led to fascinating observations sparking new interest in the role of weather and climate in infectious disease dynamics (Lipp et al., 2002). Climate constrains the range of infectious diseases, while weather affects the timing and intensity of outbreaks (Dobson \& Carper, 1993). A link was observed between rainfall and the occurrence of cholera in the city of Douala, Cameroon (Guévart et al., 2010) but no statistical measure of the relationship was provided.

In the Equatorial Monsoon climate zone of Cameroon, which includes the city of Douala, a Spatial Autoregressive Poisson regression model was used (Ngwa et al., 2016-a) to identified statistically significant associations between the risk of transmission and several environmental factors, including the presence of major water body, highway, as well as the average daily maximum temperature and precipitation levels over the preceding two weeks. The synergistic effect of sunshine hours and temperature in cholera outbreaks was demonstrated using monthly means time series data for cholera in Matlab in Bangladesh (Islam et al., 2009). A study of the influence of rainfall and temperature on the appearance and increase in the number of cases of cholera outbreak in Lusaka Zambia using an explicative model and time series analysis found that all epidemics showed a seasonal trend coinciding with the rainy season (November to March) and attributed 4.9\% of the risks to temperature and $2.4 \%$ to rainfall (Fernadez et al., 2009). Another study in Kolkata India (WHO, 2011) examined the relationship between temperature $\left({ }^{\circ} \mathrm{C}\right)$, rainfall $(\mathrm{mm})$, and relative humidity $(\%)$ and occurrence of diarrhoea and cholera using 10 years (1999-2008) retrospective data. Using univariate descriptive analysis of the collected data followed by univariate and bivariate time series analysis and ARIMA modeling, they found that some relationships between the outcome variables and the predictor variables might exist but could neither determine nor explained the exact nature of the relationships due to some important limitations of this retrospective study.

A study on the impact of climate variability on health in the Far North of Cameroon (Kometa et al., 2013) revealed a strong positive correlation between changes in rainfall, temperature, humidity and the incidence of vector-borne diseases particularly cholera and meningitis but did not provide an appropriate statistical correlation analysis. Still, in the Sudano-Sahelian climate subzone of Cameroon statistically significant relationships were found between average daily maximum temperature and rainfall levels and cholera transmission with a two weeks lack (Ngwa et al., 2016-b). However, the relative contribution of each of the weather parameters (Rainfall and temperature) to the occurrence of cholera in the City of Maroua is poorly understood. Likewise, we found no study that has quantified the relation between relative humility and the incidence of cholera in the City of Maroua. As such, gaps in knowledge exist as to the relative contribution of rainfall, temperature, and relative humidity in the City of Maroua. This study addresses this gap and examined the relationship between rainfall, temperature and relative humidity and cholera 
incidence in the city of Maroua, Far North Cameroon. In other words, the study intended to answer the following questions: How do rainfall, temperature and relative humidity and cholera incidence correlate in the city of Maroua and what is the individual contribution of each weather parameter to the incidence of cholera? Which of rainfall, temperature and relative humidity has the strongest influence on cholera incidence in the city of Maroua? Answers to these questions could help better understand the disease mechanisms in this sahelian city with implications on the operational planning public health interventions strategies. The paper has therefore two main objectives: 1) examine the individual contribution of rainfall, temperature and relative humidity to the occurrence of cholera; 2) examine the combined contribution of all three parameters and the effect of interaction between them.

To explore the relationships between the response and explanatory variables, Generalized Additive Models (GAM) have provided a flexible framework of modelling (Osei et al., 2012). The study of the geographical distribution of disease incidence and its relationship to potential risk factors has provided, and continues to provide, rich ground for the application and development of statistical methods and models (Bailey, 2001). As emphasized by Haidu (2016), the use of statistical techniques in this paper contributes to the development of research in Technical Geography.

\section{THE STUDY SETTING AND DATA}

The study is conducted in the city of Maroua, the capital city of the Far North Region of Cameroon (Fig. 1). It lies between latitude $10^{\circ} 35^{\prime} 50^{\prime \prime} \mathrm{N}$ and $10^{\circ} 35^{\prime} 50.0^{\prime \prime} \mathrm{N}$ and longitude $14^{\circ} 18^{\prime} 57^{\prime \prime} \mathrm{E}$ and $14^{\circ} 18^{\prime} 57.0^{\prime \prime} \mathrm{E}$. The average altitude in the city is $423 \mathrm{~m}$ with a surface area of 466500 ha $\left(4665 \mathrm{~km}^{2}\right)$. The total population was estimated in 2010 at about 215000 inhabitants (BUCREP, 2010). The climate is Sudano-Sahelian and the average annual temperature is $28^{\circ} \mathrm{C}$ (temperatures reach their highest levels between January and May), with actual temperatures fluctuating throughout four distinct seasons. There is one dry season and one wet season. These are further broken down based on average temperatures, yielding four distinct periods in the area: dry and relatively cool from November to January as the region experiences a shade of winter as one moves further north, dry and hot from January to April, torrentially rainy from April to June, and relatively cool and sporadically wet from June to November (IRD-MINRESI-INC, 2000).

The weekly cholera epidemiological data from 1996 to 2011 were obtained from the Regional Delegation of Public Health for the Far North. The data for all years were collected through health facility based on passive public surveillance systems, which turns active during outbreaks. In brief, during outbreaks, cholera data are collected at health facilities and sent to health districts where they are compiled and sent to the regional monitoring service (Regional Public Health Delegation) in paper format (Ngwa et al., 2016b). The cholera clinical case data used in this study were obtained at the Regional Public Health Delegation for the Far North. The weekly epidemiological incidence data for Maroua Urban Health District was extracted from the regional database and aggregated to a monthly time series data. Average monthly rainfall $(\mathrm{mm})$, average monthly air temperature (degree Celsius), and average monthly relative humidity (percent) data from 1996 to 2011 were obtained from the meteorological services (1996 to 2005) and the Institute of Agronomic Research in Maroua (2007-2011). These two dataset were merged to form a 
single weather time series data. Missing values were completed by computing the average between the previous and the following month's data.

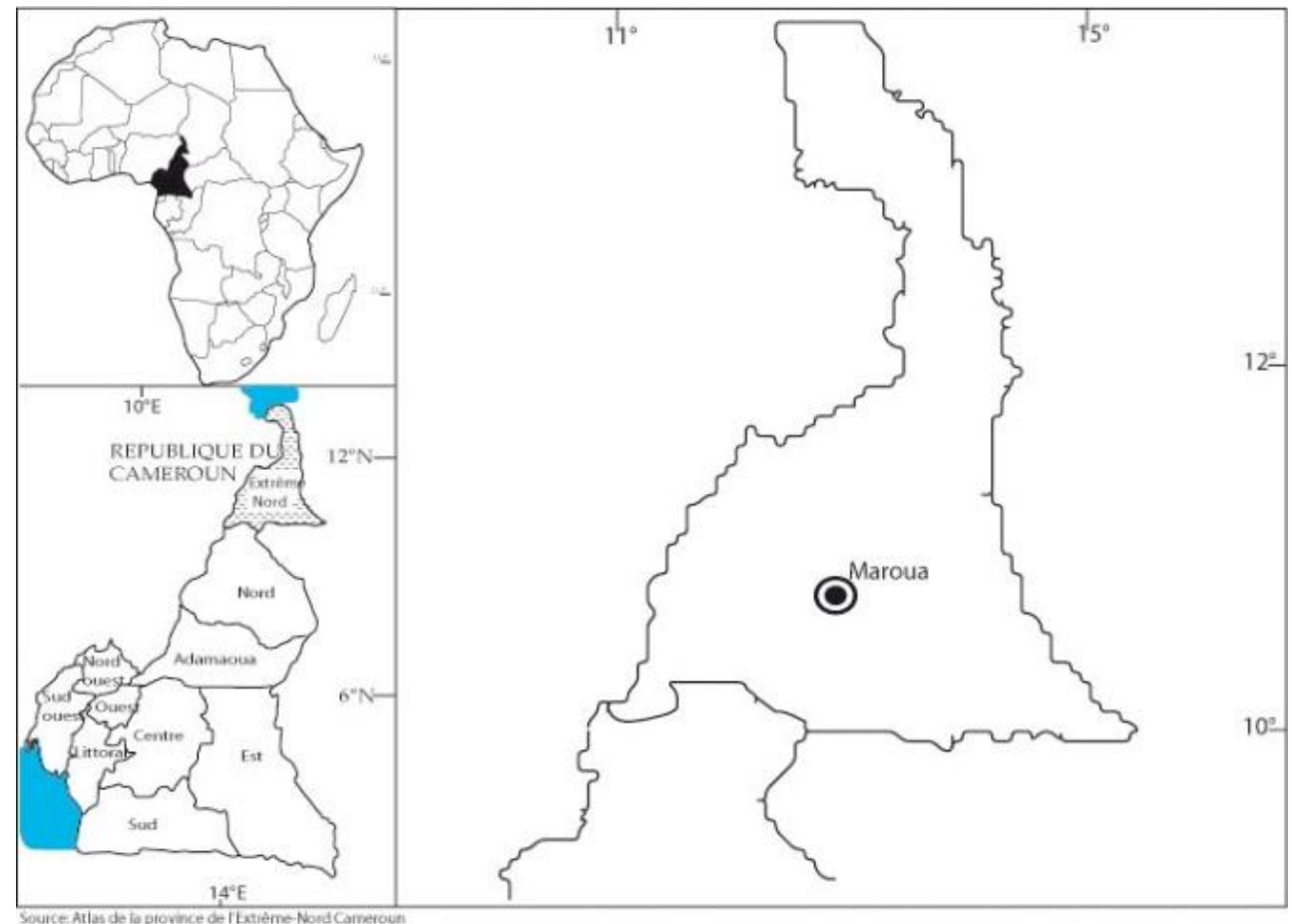

Fig. 1. Location of the study site.

\section{METHODOLOGY}

\subsection{Statistical analyses}

Simple descriptive statistics are used to capture the incidence of cholera in the city of Maroua. Total number reported cases, periods of occurrence, and various waves are discussed, the mean, standard deviation, median, minimum, maximum, range, skewness, kurtosis test and standard error of various time series were explored.

\subsection{Modeling}

The Generalized Additive Modeling (GAM) procedures were implemented to measure the contribution of each weather parameter as well as the combined contribution of all three parameters to the incidence of cholera. The GAM was used because of its ability to deal with highly non-linear and non-monotonic relationships between the response and the set of explanatory variables (Guisan et al., 2002) as it is the case in the context of this study. Furthermore, (GAM) models provide a powerful class of models for modeling nonlinear effects of continuous covariates in regression models with non-Gaussian responses (Osei et al., 2012). A GAM takes into account an additive relation between the response and predictors of a model and rules out any linearity assumption (Hastie \& Tibshirani, 1990). A series of stepwise regression models were performed between rainfall, temperature and relative humidity, and the incidence of cholera (Chatfield, 1975). The GAM model is used 
in this study to estimate the independent contribution of each rainfall, temperature and relative humidity and determined the effect of the interaction between these variable to cholera incidence in the city of Maroua.

\subsubsection{Model fitting}

Univariate GAM models were fitted on a stepwise procedure using thin plate regression spline to measure and determine separately the individual effect of humidity, temperature and rainfall on cholera incidence. Two multivariate GAMs were also fitted on a stepwise procedure to measure and determine the contribution of associated and interacting weather parameters. The first regression model was fitted to measure and determine the contribution of the association of "humidity against temperature", "humidity against rainfall", "temperature against rainfall" and "humidity against temperature vs rainfall, respectively. The second model repeated the same association but added the interaction term between the three weather parameters to the association.

\subsubsection{Evaluating the contribution of each weather parameter}

Step-wise procedures were used to evaluate the contribution of each weather parameter and identify which one influences cholera incidence the most, that is, the GAM that best describes cholera incidence. Single variable GAMs were first run separately for each of the three variables and the result was ranked in order of deviance explained. Separate univariate GAMs were therefore produced first to measure the individual relationship between cholera incidence and rainfall, cholera incidence and temperature, and cholera incidence and humidity. All the analysis and modeling process were done using the $\mathrm{R}$ studio computing software version097.551 (RStudio Team, 2012). The following functions were applied: "summary, ggplot2, mgcv, vis.gam". We chose R because it is freely available open source software.

\subsubsection{Measuring the combined contribution of three weather parameters}

At the second stage, GAMs were produced to measure the combined effect of the three weather parameters on cholera incidence considering the interaction between them. Non-parametric relationships between response and predictor variables were expressed in terms of smooth functions developed using thin plate regression splines. In this study, we used an iterative process combining the penalized thin plate regression splines because they tend to give the best Minimum Square Error (MSE) performance (Wood, 2006). In addition, the tensor product smooths often perform better than isotropic smooths when the covariates of a smooth are not naturally on the same scale. As such, their relative scaling is arbitrary (Wood, 2003). Different combinations of bivariate GAMs were later sequentially run for all three variables first with simple association (Y 1) and then with association and interaction term (Y 2) following:

$$
\begin{aligned}
& Y 1=G A M(Y \sim S(X 1)+S(X 2)) \\
& Y 2=G A M(Y \sim S(X 1)+S(X 2)+S(X 3)+T E(X 1, X 2, X 3)
\end{aligned}
$$

Where " $Y$ " represents the number of cholera cases reported; " $S$ " represents the smoother which exists purely to help set up the model using spline based smooths; "X1", "X2", and "X3" represent rainfall, relative humidity and temperature, respectively; and " $T E$ " the tensor smoother, which exists purely to help set up the model using tensor product based smooths. 
The result was ranked in order of deviance explained. Then each of the remaining variables was added in turn to the two variables model and the deviance explained recalculated. Each variable was checked with a p-value at $0.1 \%$ significance level. The sensitivity of results to changes in parameters in the smoothing process was also checked. The residuals were checked to confirm that their distribution was approximately normally distribution with zero mean and that they exhibited no clear relationship with the predictor variables or fitted values. The Durbin-Watson test statistic was used to test the independence of GAM residuals.

\subsubsection{Model selection}

The selection of the model is important, as under-fitting a model may not capture the true nature of the variability in the outcome variable, while an over-fitted model loses generality (Snipes \& Taylor, 2014). In this study the model selection was based on the Akaike Information Criterion (AIC), (Akaike, 1973) as a way to compare different models on a given outcome. The chosen model is the one that minimizes the Kullback - Leibler distance between the model and the truth defined as:

$$
A I C=-2(\ln (\text { likelihood }))+2 K
$$

Where likelihood is the probability of the data given a model and $\mathrm{K}$ is the number of free parameters in the model. AIC scores are shown as $\triangle \mathrm{AIC}$ scores, or difference between the best model (smallest AIC) and each model (so the best model has a $\triangle \mathrm{AIC}$ of zero) (Burnham \& Anderson, 2002). To assess statistical significance of the derivative (the rate of change), we examined the asymptotic normality and the point wise $95 \%$ confidence interval.

\section{RESULTS AND DISCUSSION}

\subsection{Descriptive statistics}

From 1996 to 2011, the city of Maroua was mainly hit by two waves of cholera outbreaks during this period, a total of 2259 cases of cholera were reported. The first wave lasted from July 1996 to October 1998 (Fig. 2) and the second occurred from august 2010 to November 2011 (Fig. 3).The outbreaks occurred mainly between July and November and the peaks of cases were generally recorded in August and (Fig. 2 \& 3). The characteristics of the data used are shown in Table $\mathbf{1}$.

Table 1.

Characteristics of the weather time series variables used in the city of Maroua.

\begin{tabular}{|l|l|l|r|r|r|r|r|r|r|l|}
\hline & mean & sd & \multicolumn{1}{l|}{ median } & trimmed & min & \multicolumn{1}{l|}{ max } & range & skew & kurtosis & se \\
\hline Cholera case & 11,77 & 52,48 & 0 & 0,05 & 0 & 542 & 542 & 6,85 & 57 & 3,79 \\
\hline temperature & 29,61 & 3,98 & 29 & 29,39 & 20,33 & 42,8 & 42,8 & 0,55 & 0,36 & 0,29 \\
\hline rainfal & 67,53 & 88,41 & 22,5 & 51,28 & 0 & 325,5 & 325,5 & 1,22 & 0,31 & 6,38 \\
\hline humidity & 48,02 & 22,81 & 43,83 & 47,74 & 11,75 & 86,1 & 86,1 & 0,12 & $-1,55$ & 1,65 \\
\hline
\end{tabular}




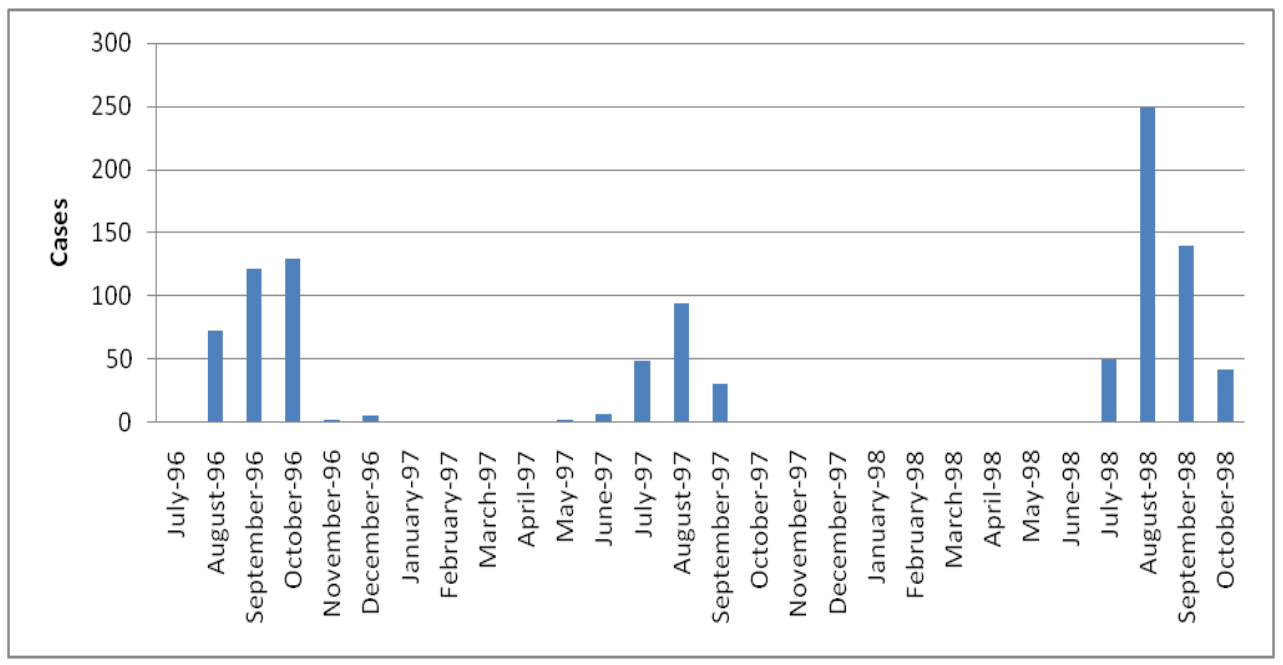

Fig. 2. Monthly number of cases in Maroua from March 1996 to May 1998.

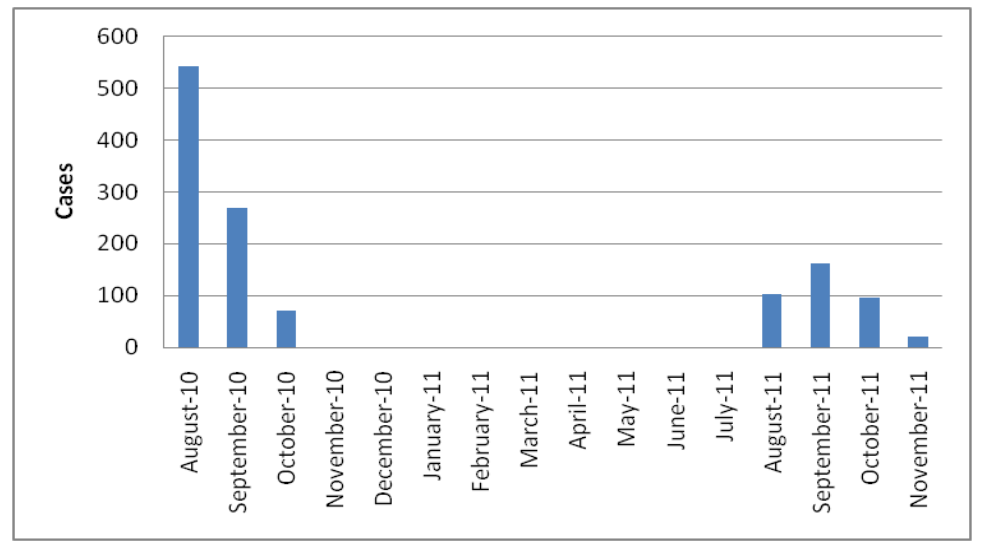

Fig. 3. Monthly number of cases in Maroua form August 2010 to November 2011.

\subsection{The effect of individual weather parameters}

As shown in Table 2 below that summarizes the results from each of the models, the $\mathrm{R}^{2}$ (coefficient of determination) and the deviance explained of temperature are the highest (0.243) and (26.8\%) respectively; AIC (2021.273) of temperature is the lowest. We note from the Table 2 above that all the three parameters are correlated with cholera incidence with temperature being the most determinant parameter. It has the highest deviance explained (26.8\%). In the results of the individual GAM models shown as plots (Fig. 4, 5, 6) for the effect of the weather parameters on cholera, the broken lines represent point-wise 95-percent confidence envelopes around the fit. We observed that cholera cases mainly occurred when temperatures are between $21^{\circ}$ and $30^{\circ}$ (Fig. 4) and relative at humidity 
between 79 and 82 (Fig. 5). The curve in Fig. 5 below shows multiple inflections for rainfall, which would suggest the effects of temperature that interacts strongly over rainfall. When associating cholera incidence with weather variables separately, we noted that the correlation is positive for all the three parameters. However, both the correlation $(0.243)$ and the deviance explained $(26.8 \%)$ by temperature are higher than the correlation with humidity (0.176) and the deviance explained (19.9\%) by humidity alone which are very weak and the correlation with rainfall (0.0644) and the deviance explained $(13.6 \%)$ by rainfall alone which are the least important. Yet in Zambia, the attributable risks were $4.9 \%$ for temperature and $2.4 \%$ for rainfall (Fernandez et al., 2009).

Performance of the weather parameters models.

Table 2.

\begin{tabular}{|l|l|l|l|l|l|}
\hline & Std error & R 2 & Deviance explained & p-value & AIC \\
\hline Humidity & 3.438 & 0.176 & $19.9 \%$ & $1.09 \mathrm{e}-06$ & 2036.569 \\
\hline temperature & 3.295 & 0.243 & $26.8 \%$ & $2.03 \mathrm{e}-09$ & 2021.273 \\
\hline Rainfall & 3.593 & 0.0644 & $13.6 \%$ & 0.00265 & 2055.812 \\
\hline
\end{tabular}

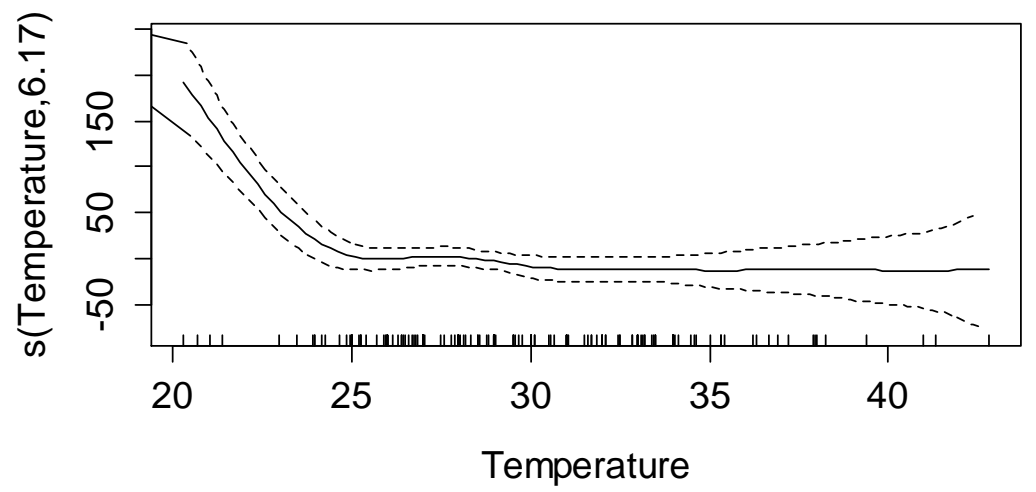

Fig. 4. The effect of temperature on cholera incidence.

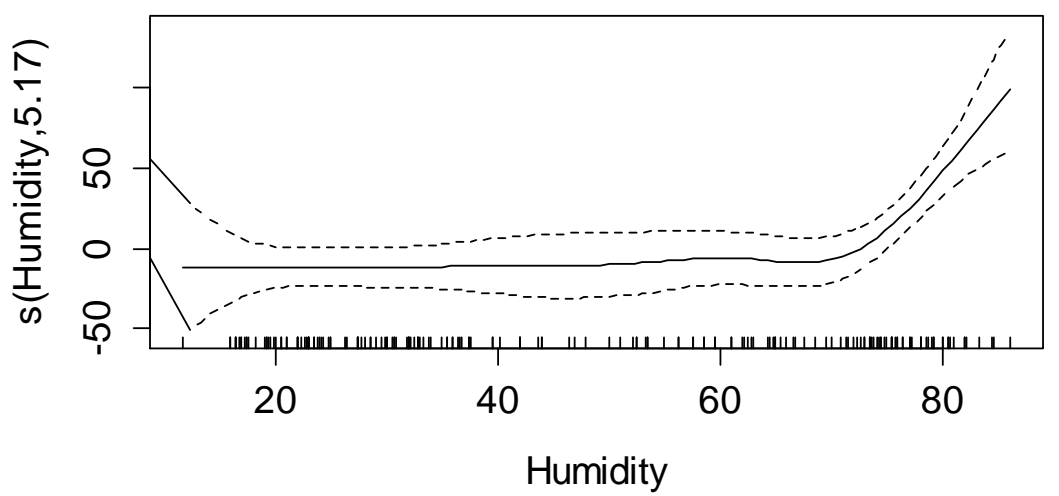

Fig. 5. The effect of humidity on cholera incidence. 


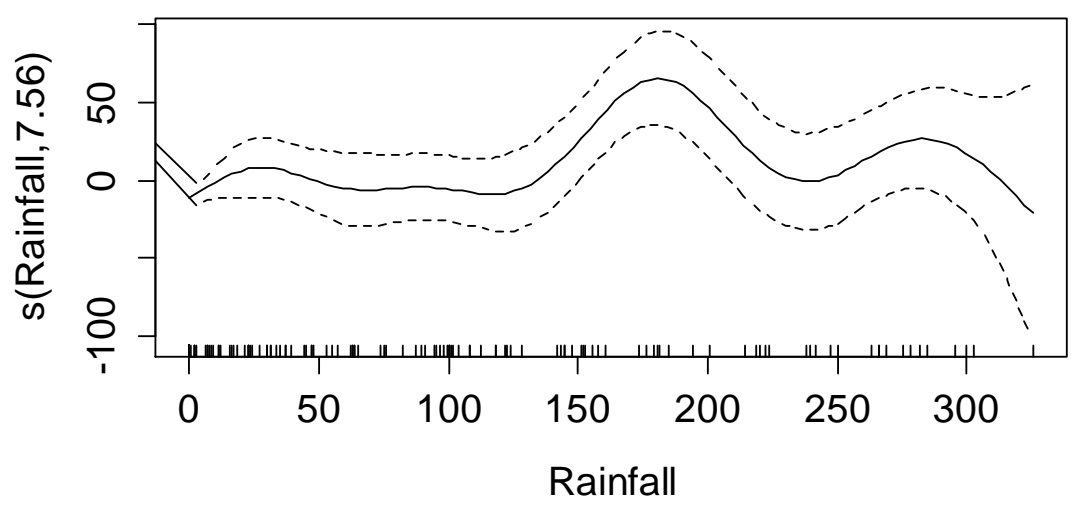

Fig. 6. The effect of rainfall on cholera incidence.

\subsection{The effect of associated weather parameter}

\section{Model 1: Using tensor smooth without interaction}

Table 3 below summarizes the results of the first set of GAM models that considered only the association of the weather parameters. From table 3 below we note that among the association of two weather parameters, "temperature against rainfall" has the highest deviance explained, the lowest generalized cross-validation (GCV) and AIC but the highest $\mathrm{R}^{2}$. This means a very strong rainfall-temperature interaction. The association of all the three parameters yields the highest deviance explained and the highest $\mathrm{R}^{2}$, but the lowest $\mathrm{AIC}$ and GCV.

Table 3.

Estimates of the GAM without interaction.

\begin{tabular}{|l|r|r|r|r|r|}
\hline & std error & R 2 & DevExp & F & \multicolumn{1}{l|}{ AIC } \\
\hline Humidity + Temperature & 3,036 & 0,358 & 43,6 & 5,238 & 2000,22 \\
\hline Humidity + Rainfall & 3,048 & 0,353 & 40,8 & 28,41 & 2000,771 \\
\hline Temperature + Rainfall & 2,504 & 0,563 & 60,4 & 12,92 & 1926,857 \\
\hline Humidity + Temperature + Rainfall & 1,264 & 0,889 & 94,1 & 816029 & 1704,733 \\
\hline
\end{tabular}

\section{Model 2: Using tensor smooth with interaction}

The results presented in table 4 below are from the second set of GAM models that takes into account both the association of weather parameters and the interaction between them. All variables selected in the final model were statistically significant. The couple "temperature vs. rainfall" still gives evidence of strong interaction according to R2 and Deviance explained. The results presented in table 4 below are from the second set of GAM models that takes into account both the association of weather parameters and the interaction between them. All variables selected in the final model were statistically significant. One can observed that the $\mathrm{R}^{2}$ and the deviance explained are higher while the 
standard error, the GVC and the AIC are lower than the first model. The couple "temperature vs. rainfall" still gives evidence of strong interaction.

Table 4.

Estimates of the final GAM with association and interaction.

\begin{tabular}{|l|r|r|r|l|}
\hline & std error & R 2 & DevExp & AIC \\
\hline Humidity + Temperature & 2,987 & 0,378 & 45,5 & 1999,331 \\
\hline Humidity + Rainfall & 2,815 & 0,448 & 52,6 & 1979,349 \\
\hline Temperature + Rainfall & 2,395 & 0,6 & 64,4 & 1911,963 \\
\hline Humidity + Temperature + Rainfall & 1,128 & 0,911 & 95,4 & 1661,502 \\
\hline
\end{tabular}

Interaction effects between the parameters are shown as perspective plots (Fig. 7) to see how the linear predictor or expected response varies with two predictors, if all the others were held fixed at some value. The y-axis reflects the relative importance of each parameter of the model, and for the interaction effects, this is presented on the z-axis. The perspective plot views of the GAM show the results of the best-fitting smooths for the variables included in the model. The interaction between the two variables is presented as a surface; the $\mathrm{z}$-axis shows the response and the relative importance of each variable is presented in the $\mathrm{x}$ - and $\mathrm{y}$ - axis. We observe from the perspective plots that the response remain at 0 producing no effect for " humidity against rainfall"; a little positive response is observed for " humidity and temperature" and a higher response is observed for the asssociation between rainfall and temperature (Fig. 7).

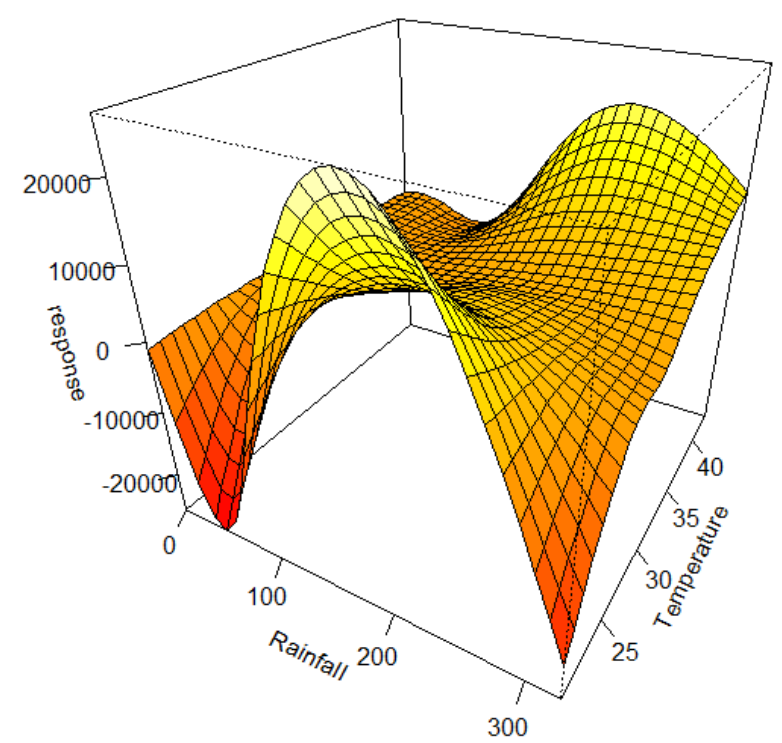

Fig. 7. The interaction betweem rainfall and temperature. 
When we consider the association between two weather parameters, the association and interaction between rainfall and temperature has a higher correlation $\mathrm{R}^{2}(0.6)$ and deviance explained (64.4\%) than the association and interaction between the other parameters. The importance of rainfall as a driver of the seasonal cycle of cholera is implied by its waterborne transmission, the dose-dependent nature of infection, and the decline of cases during the monsoon season (Pascual et al., 2002). However, warm temperatures associated with the presence of water bodies favor the growth of Vibrio cholerae (Lipp et al., 2002) and that is due to the fact that higher temperature favors the growth of phytoplankton (Patz et al., 2005; Steffen, 2005). The correlation coefficient $\mathrm{R}^{2}$ is higher (0.911) when all three parameters are simultaneously associated with $95.4 \%$ of deviance explained when considering interaction. Similar results were found in Sinazongwe District of Southern Zambia (Phiri et al., 2015) where they noted that increased precipitation was associated with the occurrence of cholera outbreaks with a Spearman rank correlation $\mathrm{r}=.86$ between the number of cholera cases and the amount of precipitation (Spearman $\mathrm{r}=0.86 ; \mathrm{P}<.01$ ). On the other hand, $V$. cholera infection was associated with higher Relative Humidity $(>80 \%)$ at $29^{\circ} \mathrm{C}$ temperature with intermittent average $(10 \mathrm{~cm})$ rainfall (Rajendran et al., 2011). Heavy rainfall indirectly influenced the $V$. cholera infection, whereas no correlation was found with high temperature.

\section{CONCLUSION}

In this study we found that in Maroua city temperature has the highest coefficient of determination and that cholera is most likely to occur at temperature between $21^{\circ} \mathrm{C}$ and $35^{\circ} \mathrm{C}$ when the average monthly rainfall is above $100 \mathrm{~mm}$. The effect of humidity appears to be very negligible. The bivariate association and interaction between rainfall and temperature has a higher coefficient of determination and deviance explained. The multivariate association with interaction of all three parameters with cholera has higher values for both the deviance explained and the coefficient of determination. These results indicate that statistical time series models should lead to a better understanding of the disease mechanism in this sahelian city, thus contributing to the growing debate on the relationship between weather parameter sand the occurrence of cholera.

\section{ACKNOWLEDGEMENTS:}

Mouhaman Arabi was supported in part by the University of Maroua and Moise Chi Ngwa was supported in part by the DOVE-Project at Johns Hopkins Bloomberg School of Public Health.

\section{R E F E R E N C E S}

Akaike, H. (1973). Information theory and an extension of the maximum likelihood principle. In B.N. Petrov., and F. Csaki (Eds.), Second International Symposium on Information Theory. Budapest: Symposium, Academia Kiado.

Ali, M, Nelson, A.R., Lopez, A.L., Sack, D. (2015). PLoS Neglected Tropical Diseases 9(6): e0003832. doi:10.1371/journal.pntd.0003832.

Bailey, T.C. (2001). Spatial statistical methods in health. Cadernos de Saúde Pública. 17(5), 10831098. https://dx.doi.org/10.1590/S0102-311X2001000500011. 
BUCREP (2010) La population du Cameroun en 2010.

http://www.bucrep.cm/index.php/en/?option=com_phocadownload\&view=file\&id=18:populati on-du-cameroun-en-2010\&Itemid=39\&lang=fr.

Burnham K. P., \& Anderson D. R. (2002) Model Selection and Multi-model Inference A Practical Information-Theoretic Approach, Second Edition, Springer-Verlag, New York.

Chatfield C. (1975) The analysis of time series: theory and practice. Chapman \& Hall; New York, London.

Codeço CT, Coelho FC (2006) Trends in cholera epidemiology. PLoS Medicine 3(1): e42 10.1371/journal.pmed.0030042.

Dobson, A. Carper, R., (1993) Biodiversity, Lancet, 342, 1096-1099.

Fernandez, L., Bauernfeind, A., Jimenez, J.D., Gill, C.L, El Omeiri, N and Guilbert, D.H. (2009) Influence of temperature and rainfall on the evolution of cholera epidemics in Lusaka, Zambia, 2003-2006: analysis of a time series. Trans R Soc Trop Med Hyg., 103(2), 137-43, DOI: 10.1016/j.trstmh.2008.07.017.

Guévart E, Noeske J, Gérémie SG, Fouda AB, Mouangue A, Manga B. (2010) Weather and cholera: epidemic in Douala, Cameroon in 2004. Médecine tropicale: revue du Corps de santé colonial 70(4),407-8

Guisan, A., Edwards, T.C-Jr., \& T. Hastie. (2002). Generalized linear and generalized additive models in studies of species distributions: setting the scene. Ecological Modelling, 157(2-3), 89100.

Haidu, I. (2016). What Is Technical Geography. Geographia Technica, 11(1), 1-5. DOI: 10.21163/GT_2016.111.01

Hastie, T. \& Tibshirani, R. (1990). Generalized Additive Models. Monographs on Statistics \& Applied Probability, Chapman \& Hall.

IRD-MINRESI/INC (2000) Atlas de la province extrême-nord Cameroun ; IRD Éditions.

Islam, M.S., Sharker, M.A., Rheman S., Hossain S., Mahmud Z.H., Islam M.S., Uddin A.M., Yunus M., Osman M.S., Ernst R., Rector I., Larson C.P., Luby S.P., Endtz H.P., Cravioto A. (2009) Effects of local climate variability on transmission dynamics of cholera in Matlab, Bangladesh. Transactions of the Royal Society of Tropical Medicine and Hygiene. 103(11) 1165-70. 10.1016/j.trstmh.2009.04.016.

Kometa, S. S., Mathias, A. T. E., Humphrey, N. N. and Amawa, S. G. (2013) Human health vulnerability to climate variability: The cases of Cholera and Meningistis in some urban areas of the far north region of Cameroon. Journal of Geography and Geology, 5(1),116-125.

McMichael, A.J., (2015) Extreme weather events and infectious disease outbreaks, Virulence, 6:6, 543-547, DOI: 10.4161/21505594.2014.975022.

Lipp, E.,. Huq, A. \& Colwell, R.R. (2002) Effects of Global Climate on Infectious Disease: the Cholera Model. American Society for Microbiology. Vol 15 No 4.

Ngwa, M.C., Liang, S., Kracalik, I.T., Morris, L., Blackburn, J.K., Mbam, L.M., Ba Pouth, S.F.B., Teboh, A., Yang, Y., Arabi, M., Sugimoto, J.D. \& Morris, J.G., (2016-a) Cholera in Cameroon, 2000-2012: Spatialand Temporal Analysis at the Operational Health District and Sub Climate Levels. PLOS Neglected Tropical Diseases. DOI:10.1371/journal.pntd.0005105.

Ngwa, M. C., Liang, S., Mbam, L. M., Mouhaman, A., Teboh, A., Brekmo, K., Mevoula, O. \& Morris, J. G., (2016-b) Cholera public health surveillance in the Republic of Cameroon opportunities and challenges. Pan African Medical Journal. 24(222).

Osei, F.B., Dulker A.A. \& Stein A. (2012) Bayesian structured additive regression modelling of epidemic data: application to cholera. BMC Medical Research Methodology. 12 (118), http://www.biomedcentral.com/1471-2288/12/118. 
Pascual M, Bouma MJ, Dobson AP (2002) Cholera and climate: revisiting the quantitative evidence. Microbes and Infection 4:237-245.

Patz, J.A., Campell-Lendrum, D., Holloway, T., \& Foley, J.A., (2005), Impact of regional climate change on hiuman health. Nature, 438(17), 310-317.

Phiri, P., Nzala, S.H, Baboo, K. S. (2015) Factors associated with the recurring cholera outbreaks in Sinazongwe, district of southern Zambia. Medical Journal of Zambia, 42(4), 184-192.

Rajendran, K., Sumi, A., Bhattachariya, M.K., Manna, B., Sur, D., Kobayashi, N. \& Ramamurthy, T., (2011) Influence of Relative Humidity in Vibrio Cholerae Infection: A Time Series Model. Indian Journal of Medical Research, 133: 138-45.

Reyburn, R., Kim, D. R., Emch, M., Khatib, A., von Seidlein, L., \& Ali, M., (2011) Climate Variability and the Outbreaks of Cholera in Zanzibar East Africa: A Time Series Analysis. American Journal of Tropical Medicine and Hygiene, 84(6), 862-869.

RStudio Team (2012). RStudio: Integrated Development for R. RStudio, Inc., Boston, MA. URL http://www.rstudio.com/

Snipes, M., \& Taylor, D. (2014) Model Selection and Akaike Information Criteria: An Example from Wine Ratings and Prices. Wine Economics and Policy. 3(1), 3-9, doi.org/10.1016/j.wep.2014.03.001.

Steffen, W. (2006) Stronger evidence but new challenges: climate chang science 2001-2005. 28 p. Canberra, A.C.T. : Australian Greenhouse Office.

WHO (2011) Relationship Between Climate Variability And Occurrence Of Diarrhoea And Cholera A Pilot Study Using Retrospective Data From Kolkata, India; Technical Report National Institute Of Cholera \& Enteric Diseases (Niced) Kolkata, India.

WHO (2012) Cholera Fact sheet $N^{\circ} 107$ available online from: http://www.who.int/mediacentre/factsheets/fs107/en/index.html.

Wood S.N. (2006) Generalized Additive Models: An Introduction with R. Chapman and Hall/CRC Press.

Wood, S.N. (2003) Thin plate regression splines. Journal Royal Statistics Society B, 65(1), 95-114. 